\title{
Smart technologies: useful tools to assess the exposure to solar ultraviolet radiation for general population and outdoor workers
}

\author{
A. Militello ${ }^{I}$, M.Borra $^{l}$, C. Grandi ${ }^{1}$ \\ Department of Occupational and Environmental Medicine, \\ Epidemiology and Hygiene \\ Italian Workers' Compensation Authority (INAIL) \\ Monte Porzio Catone (RM) - ITALY
}

\author{
F. Bisegna ${ }^{2}$ \\ ${ }^{2}$ Department of Astronautic, Electric and Energetic \\ Engineering \\ University of Rome "Sapienza" \\ Rome - ITALY
}

\begin{abstract}
The ultraviolet radiation is a key component of the solar spectrum, giving an important contribution to the overall dose collected by people exposed to solar radiation. Beside some widely and well documented benefits attributed to solar radiation, a lot of adverse effects are described, including the occurrence of photo-induced skin cancer and ocular diseases as a consequence of chronic exposure to ultraviolet radiation; the International Agency for Research on Cancer (IARC) classifies the solar spectrum as a whole and the individual spectral bands UVA, UVB and UVC as "carcinogenic to humans" (group 1 of the IARC classification of the carcinogenic evidence).
\end{abstract}

An improvement of risk perception on risks due to ultraviolet radiation overexposure in the population currently exposed to solar radiation, both occupationally and recreationally, is of great importance by a public health point of view. In parallel, the amount of exposure to ultraviolet radiation has to be assessed as accurately as possible, with the aim to identify and characterize different exposure situations and, by their appropriate management, to prevent adverse health effects attributed to prolonged exposure to solar radiation.

The available technology allows to acquire such information, either using miniaturized and wearable sensors, or through devices with applications exploiting the integration between satellite-based or meteorological data on ultraviolet irradiance and geolocation (e.g. App on smartphones). The diffusion of such devices could be extended, provided that these tools doesn't require specific skills for the users and that it becomes not necessary a post-processing of the data (if not for research purposes), together with a low purchase cost and a reliability and ease of use.

The aim of this paper is to evaluate the performance of some available devices, comparing the information provided by the last ones with that obtained by the use of an ad hoc scientific instrumentation, also in order to stimulate the producers' interest in the development of tools with specific requirements and functionality. The information thus obtained may contribute to a better definition in quantitative terms of the cause-effect relationship in epidemiological studies on photo-induced skin and ocular diseases Moreover, individual exposure data should be useful for occupational exposure management and health surveillance practice in outdoor workers. More generally, they may support general practitiones to address patients toward the adoption of personal preventive and protective measures and to follow healthier lifestyles.

Keywords- Solar radiation; ultraviolet radiation; smart technologies; public health; outdoor worker 